\title{
Mathematical Model for Zika Virus Dynamics with Sexual Transmission Route
}

\author{
F.B. Agusto ${ }^{\dagger *}$ S. Bewick ${ }^{\ddagger}$ and W.F. Fagan ${ }^{\ddagger}$ \\ ${ }^{\dagger}$ Department of Ecology 63 Evolutionary Biology, University of Kansas, Lawrence KS, 66045

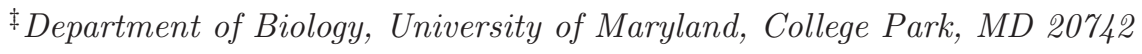

December 6, 2016

\footnotetext{
*Email: fbagusto@gmail.com
} backward bifurcation

\section{Introduction} discussions worldwide [54].

\begin{abstract}
Zika virus is a flavivirus transmitted to humans primarily through the bite of infected Aedes mosquitoes. In addition to vector-borne spread, however, the virus can also be transmitted through sexual contact. In this paper, we formulate and analyze a new system of ordinary differential equations which incorporates both vector and sexual transmission routes. Theoretical analysis of this model when there is no disease induced mortality shows that the disease-free equilibrium is locally and globally asymptotically stable whenever the associated reproduction number is less than unity and unstable otherwise. However, when we extend this same model to include Zika induced mortality, which have been documented in Latin America, we find that the model exhibits a backward bifurcation. Specifically, a stable disease-free equilibrium co-exists with a stable endemic equilibrium when the associated reproduction number is less than unity. To further explore model predictions, we use numerical simulations to assess the importance of sexual transmission to disease dynamics. This analysis shows that risky behavior involving multiple sexual partners, particularly among male populations, substantially increases the number of infected individuals in the population, contributing significantly to the disease burden in the community.
\end{abstract}

2000 Mathematics Subject Classifications: 92B05, 93A30, 93C15.

Keywords: Zika virus; heterosexual and homosexual couples; sexual transmission; stability;

Historically confined to Africa and Southeast Asia [49, 18, 29], the recent introduction of Zika virus (ZIKV) into Latin America [68] has resulted in alarming progression [59] of this disease across much of the New World. Indeed, as of May 26, 2016, only two countries in South America - Chile and Uruguay - had not yet reported autochthonous spread of Zika [14]. Unfortunately, the emergence of Zika under these new conditions has brought with it new discoveries of troubling viral outcomes, including Guillan Barre syndrome [47, 10] and microcephaly [43, 48] in adults and newborns respectively. With the novel realization that Zika can cause severe complications, a disease once described as a 'mild form of dengue' has come to the forefront of public health 

that half of these are females. In this case, the rate at which females are born is given by $\Pi_{F}=\frac{\Pi_{H}}{2}$. At the same time, the female population is reduced as a result of natural death at a 
rate $\left(\mu_{F}\right)$. The total number of humans and mosquitoes in the community determines the average number of mosquito bites a human will receive. Here, we assume that the mosquito biting rate, $b_{V}$, is constant, and represents the maximal rate allowed by the gonotrophic cycle [67]. Let $b_{H}$ be the rate at which bites are received by a single human. For the number of bites to be conserved, the total number of bites by mosquitoes must be equal to the total number of bites received by humans. Hence,

$$
b_{V} N_{V}=b_{H}\left(N_{H}, N_{V}\right) N_{H}
$$

so that,

$$
N_{V}=\frac{b_{H}\left(N_{H}, N_{V}\right) N_{H}}{b_{V}}
$$

Let $b_{H} \beta_{V}$ be the effective contact rate between a susceptible human and infectious mosquitoes, where $\beta_{V}$ is the transmission probability per contact from an infectious mosquito to a susceptible human. Also, let $b_{V} \beta_{V}$ be the effective contact rate between a susceptible mosquito and infectious human, where $\beta_{V}$ is the transmission probability per contact from an infectious human to a susceptible mosquito. In this case, susceptible females acquire infection, following effective contact with an infectious mosquito, at a rate $\lambda_{F 1}$, given by,

$$
\lambda_{H}\left(I_{V}, N_{H}\right)=\frac{b_{H}\left(N_{F}, N_{V}\right) \beta_{H} I_{V}}{N_{V}} .
$$

Substituting (2.1) in (2.2) we obtain,

$$
\lambda_{H}\left(I_{V}, N_{H}\right)=\frac{b_{V} \beta_{H} I_{V}}{N_{H}} .
$$

It can also be shown that the rate at which mosquitoes acquire infection from infectious humans is given by

$$
\lambda_{V}\left(I_{F}, I_{M}, N_{H}\right)=\frac{b_{V} \beta_{V}\left(I_{F}+I_{M}\right)}{N_{H}} .
$$

The parameter $\beta_{V}$ is the probability that a bite from a susceptible mosquito to a human will lead to infection of the mosquito.

For sexual transmission, we assume that the infection rate of susceptible females by infected males $\left(\lambda_{F M}\right)$ is given as:

$$
\lambda_{F M}\left(I_{M}, N_{M}\right)=\frac{C_{F} \beta_{M} I_{M}}{N_{M}}
$$

where the parameter $\beta_{M}$ is the probability that a sexual contact with an infectious male will lead to infection of the susceptible female and the parameter $C_{F}$ is the contact rate between males and females. It is assumed that the contact rate is constant. Thus, for the number of contact to be conserved, the total number of contacts by males $\left(C_{M_{1}}\right)$ must be equal to the total number of contacts by females $\left(C_{F}\right)$,

$$
C_{M_{1}} N_{M}=C_{F} N_{F}
$$

so that,

$$
N_{M}=\frac{C_{F} N_{F}}{C_{M_{1}}}
$$


Using equation (2.4) in equation (2.3) we obtain,

$$
\lambda_{F M}\left(I_{M}, N_{F}\right)=\frac{C_{M_{1}} \beta_{M} I_{M}}{N_{F}} .
$$

We do not consider sexual transmission between female partners, since this is extremely rare in other sexually transmitted diseases, and thus is not expected to occur for Zika either [51].

Hence, susceptible females become infected following effective contact with infected mosquitoes at a rate $\lambda_{F 1}\left(I_{V}, N_{H}\right)$ and from sexual contact with infected males at a rate $\lambda_{F M}\left(I_{M}, N_{F}\right)$ after which they move to the exposed class. This gives

$$
\frac{d S_{F}}{d t}=\Pi_{F}-\frac{b_{V} \beta_{H} I_{V} S_{F}}{N_{H}}-\frac{C_{M_{1}} \beta_{M} I_{M} S_{F}}{N_{F}}-\mu_{F} S_{F} .
$$

Exposed females move to the infectious class due to disease progression at a rate $\sigma_{F}$. The population of exposed females is decreased due to natural death at a rate $\mu_{F}$. Thus, the equation for the exposed class is represented by

$$
\frac{d E_{F}}{d t}=\frac{b_{V} \beta_{H} I_{V} S_{F}}{N_{H}}+\frac{C_{M_{1}} \beta_{M} I_{M} S_{F}}{N_{F}}-\left(\sigma_{F}+\mu_{F}\right) E_{F} .
$$

Likewise, the infected class population is decreased as a result of recovery at a rate $\gamma_{F}$ and natural death at a rate $\mu_{F}$. Hence, we have

$$
\frac{d I_{F}}{d t}=\sigma_{F} E_{F}-\left(\gamma_{F}+\mu_{F}\right) I_{F} .
$$

Finally, the population of recovered individuals is comprised of individuals that have progressed through the infectious stage and become refractory to further diseases. This population is then decreased due to natural death at a rate $\mu_{F}$. Thus, the equation for the recovered population becomes:

$$
\frac{d R_{F}}{d t}=\gamma_{F} I_{F}-\mu_{F} R_{F}
$$

The equations for the male sub-population are similarly obtained; additionally, however, we include male-to-male transmission [17]. Although there are no reported cases of female-to-male transmission, this possibility cannot be ruled out and we assume the existence of this transmission route, though possibly with a different transmission probability per encounter.

For the vector population, we assume that susceptible mosquitoes are recruited at a rate $\left(\Pi_{V}\right)$. This population is then reduced by biting on infectious humans, and as a result of natural death at a rate $\mu_{V}$. This gives:

$$
\frac{d S_{V}}{d t}=\Pi_{V}-\frac{b_{V} \beta_{V}\left(I_{F}+I_{M}\right) S_{V}}{N_{H}}-\mu_{V} S_{V}
$$

When a susceptible mosquito bites an infectious human, then with probability of infection $\left(\beta_{V}\right)$, the mosquito move to the exposed class $\left(E_{V}\right)$. The equation for this class is given by:

$$
\frac{d E_{V}}{d t}=\frac{b_{V} \beta_{V}\left(I_{F}+I_{M}\right) S_{V}}{N_{H}}-\left(\sigma_{V}+\mu_{V}\right) E_{V}
$$


where we have again assumed a natural death rate of $\mu_{V}$. Exposed mosquitoes move to the infected class at the rate $\sigma_{V}$ and are again reduced by natural death. Thus, we have:

$$
\frac{d I_{V}}{d t}=\sigma_{V} E_{V}-\mu_{V} I_{V}
$$

Overall, our model for ZIKV, including both sexual and vector-borne transmission, is given as:

$$
\begin{aligned}
\frac{d S_{F}}{d t} & =\Pi_{F}-\frac{b_{V} \beta_{H} I_{V} S_{F}}{N_{H}}-\frac{C_{M_{1}} \beta_{M} I_{M} S_{F}}{N_{F}}-\mu_{F} S_{F} \\
\frac{d E_{F}}{d t} & =\frac{b_{V} \beta_{H} I_{V} S_{F}}{N_{H}}+\frac{C_{M_{1}} \beta_{M} I_{M} S_{F}}{N_{F}}-\left(\sigma_{F}+\mu_{F}\right) E_{F} \\
\frac{d I_{F}}{d t} & =\sigma_{F} E_{F}-\left(\gamma_{F}+\mu_{F}\right) I_{F} \\
\frac{d R_{F}}{d t} & =\gamma_{F} I_{F}-\mu_{F} R_{F} \\
\frac{d S_{M}}{d t} & =\Pi_{M}-\frac{b_{V} \beta_{H} I_{V} S_{M}}{N_{H}}-\frac{C_{F} \beta_{F} I_{F} S_{M}}{N_{M}}-\frac{C_{M_{2}} \beta_{M} I_{M} S_{M}}{N_{M}}-\mu_{M} S_{M} \\
\frac{d E_{M}}{d t} & =\frac{b_{V} \beta_{H} I_{V} S_{M}}{N_{H}}+\frac{C_{F} \beta_{F} I_{F} S_{M}}{N_{M}}+\frac{C_{M_{2}} \beta_{M} I_{M} S_{M}}{N_{M}}-\left(\sigma_{M}+\mu_{M}\right) E_{M} \\
\frac{d I_{M}}{d t} & =\sigma_{M} E_{M}-\left(\gamma_{M}+\mu_{M}\right) I_{M} \\
\frac{d R_{M}}{d t} & =\gamma_{M} I_{M}-\mu_{M} R_{M} \\
\frac{d S_{V}}{d t} & =\Pi_{V}-\frac{b_{V} \beta_{V}\left(I_{F}+I_{M}\right) S_{V}}{N_{H}}-\mu_{V} S_{V} \\
\frac{d E_{V}}{d t} & =\frac{b_{V} \beta_{V}\left(I_{F}+I_{M}\right) S_{V}}{N_{H}}-\left(\sigma_{V}+\mu_{V}\right) E_{V} \\
\frac{d I_{V}}{d t} & =\sigma_{V} E_{V}-\mu_{V} I_{V} \\
&
\end{aligned}
$$

${ }_{105}$ where $\Pi_{F}=\frac{\Pi_{H}}{2}$ and $\Pi_{M}=\frac{\Pi_{H}}{2}$. 


\section{Females}

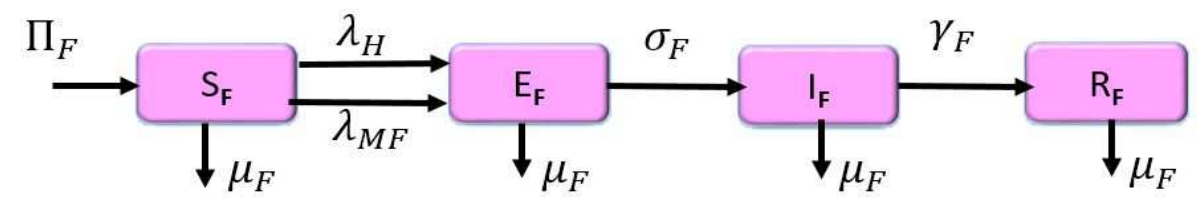

\section{Males}

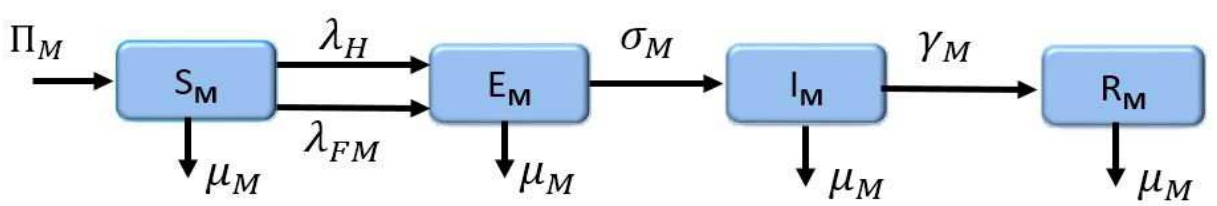

\section{Mosquitoes}

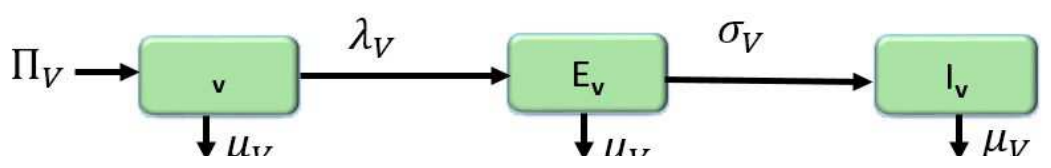

Figure 1: Flow diagram of the Zika virus model (2.5) with sexual transmission route 


\begin{tabular}{ll}
\hline \hline Variable & Description \\
\hline \hline$S_{F}(t), S_{M}(t)$ & Susceptible females and males \\
$E_{F}(t), E_{M}(t)$ & Exposed females and males \\
$I_{F}(t), I_{M}(t)$ & Infected females and males \\
$R_{F}(t), R_{M}(t)$ & Recovered females and males \\
$S_{V}(t)$ & Susceptible mosquitoes \\
$E_{V}(t)$ & Exposed mosquitoes \\
$I_{V}(t)$ & Infected mosquitoes \\
& \\
\hline \hline Parameter & Description \\
\hline \hline & \\
$\Pi_{H}$ & Recruitment rate of females and males \\
$C_{F}, C_{M_{1}}$ & Heterosexual contact rate between females and males \\
$C_{M_{2}}$ & Homosexual contact rate between males \\
$\beta_{H}$ & Transmission probability per contact of susceptible humans with infected mosquitoes \\
$\sigma_{F}, \sigma_{M}$ & Progression rate of exposed females and males \\
$\gamma_{F}, \gamma_{M}$ & Recovery rate of females and males \\
$\mu_{F}, \mu_{M}$ & Natural death rate of females and males \\
$\pi_{V}$ & Recruitment rate of mosquitoes \\
$\beta_{F}, \beta_{M}$ & Transmission probability per contact of females and males \\
$b_{V}$ & Mosquito biting rate \\
$\beta_{V}$ & Transmission probability per contact of susceptible mosquitoes with infected humans \\
$\sigma_{V}$ & Progression rate of exposed mosquitoes \\
$\mu_{V}$ & Natural death rate of mosquitoes \\
\hline \hline
\end{tabular}

Table 1: Description of the state variables and parameters of the Zika virus model (2.5) with sexual transmission route.

\subsection{Basic properties}

We shall now explore the basic dynamical features of model (2.5). Since model (2.5) describes both human and mosquito populations during the course of a Zika epidemic, it will only be epidemiologically meaningful if all state variables are non-negative for $t \geq 0$. That is, its solution with positive initial data will remain positive for all time $(t>0)$.

Lemma 1. Let the initial data $F(0) \geq 0$, where $F(t)=\left(S_{F}, E_{F}, I_{F}, R_{F}, S_{M}, E_{M}, I_{M}, R_{M}\right.$, $\left.S_{V}, E_{V}, I_{V}\right)$. Then the solutions $F(t)$ of model (2.5) are non-negative for all time $t>0$. Furthermore

$$
\limsup _{t \rightarrow \infty} N_{H}(t)=\frac{\Pi_{H}}{\mu_{H}}, \quad \limsup _{t \rightarrow \infty} N_{V}(t)=\frac{\Pi_{V}}{\mu_{V}},
$$

where $\mu_{H}=\min \left\{\mu_{F}, \mu_{M}\right\}$.

with,

$$
\begin{aligned}
N_{F}(t) & =S_{F}(t)+E_{F}(t)+I_{F}(t)+R_{F}(t), \\
N_{M}(t) & =S_{M}(t)+E_{M}(t)+I_{M}(t)+R_{M}(t), \\
N_{V}(t) & =S_{V}(t)+E_{V}(t)+I_{V}(t) .
\end{aligned}
$$

The proof of Lemma 1 is given in Appendix A. 
Model (2.5) will be analyzed in a biologically-feasible region as follows. Consider the feasible region

$$
\Gamma=\Gamma_{H} \times \Gamma_{V} \subset \mathbb{R}_{+}^{8} \times \mathbb{R}_{+}^{3}
$$

118

with,

$$
\begin{aligned}
& \Gamma_{H}=\left\{\left(S_{F}(t), E_{F}(t), I_{F}(t), R_{F}(t), S_{M}(t), E_{M}(t), I_{M}(t), R_{M}(t)\right): N_{H}(t) \leq \frac{\Pi_{H}}{\mu_{H}}\right\}, \\
& \Gamma_{V}=\left\{\left(S_{V}(t), E_{V}(t), I_{V}(t)\right): N_{V}(t) \leq \frac{\Pi_{V}}{\mu_{V}}\right\} .
\end{aligned}
$$

Lemma 2. The region $\Gamma=\Gamma_{H} \times \Gamma_{V} \subset \mathbb{R}_{+}^{8} \times \mathbb{R}_{+}^{3}$ is positively invariant for the basic model (2.5) with non-negative initial conditions in $\mathbb{R}_{+}^{11}$.

The proof of Lemma 2 is given in Appendix B.

\section{Analysis of the sub-models}

Before analyzing the full Zika virus transmission model (2.5), it is imperative to gain insights into the dynamics of the different transmission routes. Thus, we will separately consider two sub-models: one with the mosquito transmission route only and one with the sexual transmission route only.

\subsection{Mosquito transmission route only model}

The mosquito transmission route only model is obtained by assuming that Zika virus is not transmitted sexually. Hence, model (2.5) reduces to the following 


$$
\begin{aligned}
\frac{d S_{F}}{d t} & =\Pi_{F}-\frac{b_{V} \beta_{H} I_{V} S_{F}}{N_{H}}-\mu_{F} S_{F} \\
\frac{d E_{F}}{d t} & =\frac{b_{V} \beta_{H} I_{V} S_{F}}{N_{H}}-\left(\sigma_{F}+\mu_{F}\right) E_{F} \\
\frac{d I_{F}}{d t} & =\sigma_{F} E_{F}-\left(\gamma_{F}+\mu_{F}\right) I_{F} \\
\frac{d R_{F}}{d t} & =\gamma_{F} I_{F}-\mu_{F} R_{F} \\
\frac{d S_{M}}{d t} & =\Pi_{M}-\frac{b_{V} \beta_{H} I_{V} S_{M}}{N_{H}}-\mu_{M} S_{M} \\
\frac{d E_{M}}{d t} & =\frac{b_{V} \beta_{H} I_{V} S_{M}}{N_{H}}-\left(\sigma_{M}+\mu_{M}\right) E_{M} \\
\frac{d I_{M}}{d t} & =\sigma_{M} E_{M}-\left(\gamma_{M}+\mu_{M}\right) I_{M} \\
\frac{d R_{M}}{d t} & =\gamma_{M} I_{M}-\mu_{M} R_{M} \\
\frac{d S_{V}}{d t} & =\Pi_{V}-\frac{b_{V} \beta_{V}\left(I_{F}+I_{M}\right) S_{V}}{N_{H}}-\mu_{V} S_{V} \\
\frac{d E_{V}}{d t} & =\frac{b_{V} \beta_{V}\left(I_{F}+I_{M}\right) S_{V}}{N_{H}}-\left(\sigma_{V}+\mu_{V}\right) E_{V} \\
\frac{d I_{V}}{d t} & =\sigma_{V} E_{V}-\mu_{V} I_{V} .
\end{aligned}
$$

Consider the feasible region

$$
\Gamma_{M}=\Gamma_{H} \times \Gamma_{V} \subset \mathbb{R}_{+}^{8} \times \mathbb{R}_{+}^{3}
$$

130

with,

$$
\begin{aligned}
& \Gamma_{H}=\left\{\left(S_{F}(t), E_{F}(t), I_{F}(t), R_{F}(t), S_{M}(t), E_{M}(t), I_{M}(t), R_{M}(t): N_{H}(t)\right) \leq \frac{\Pi_{H}}{\mu_{H}}\right\}, \\
& \Gamma_{V}=\left\{\left(S_{V}(t), E_{V}(t), I_{V}(t)\right): N_{V}(t) \leq \frac{\Pi_{V}}{\mu_{V}}\right\} .
\end{aligned}
$$

It can be shown that all solutions of the system (3.1) starting in $\Gamma_{M}$ remain in $\Gamma_{M}$ for all $t>0$. Thus, $\Gamma_{M}$ is positively-invariant (hence, it is sufficient to consider the dynamics of (3.1) in $\Gamma_{M}$ ).

\subsubsection{Local stability of disease-free equilibrium (DFE)}

The disease-free equilibrium (DFE) of the model (3.1) is given as

$$
\begin{aligned}
\mathcal{E}_{V_{1}} & =\left(S_{F}^{*}, E_{F}^{*}, I_{F}^{*}, R_{F}^{*}, S_{M}^{*}, E_{M}^{*}, I_{M}^{*}, R_{B}^{*}, S_{V}^{*}, E_{V}^{*}, I_{V}^{*}\right) \\
& =\left(\frac{\Pi_{F}}{\mu_{F}}, 0,0,0, \frac{\Pi_{M}}{\mu_{M}}, 0,0,0, \frac{\Pi_{V}}{\mu_{V}}, 0,0\right) .
\end{aligned}
$$

The local asymptotic stability of $\mathcal{E}_{V_{1}}$ can be established using the next generation matrix method on the system (2.5). Taking the infected compartments $\left(E_{F}^{*}, I_{F}^{*}, E_{M}^{*}, I_{M}^{*}, E_{V}^{*}, I_{V}^{*}\right)$ at the DFE and using the notation in [63], the Jacobian matrices $F_{V}$ and $V_{V}$ for the new infection terms and the remaining transfer terms are respectively given by, 


$$
\left[\begin{array}{cccccc}
0 & 0 & 0 & 0 & 0 & \frac{b_{V} \beta_{H} S_{F}^{*}}{N_{H}^{*}} \\
0 & 0 & 0 & 0 & 0 & 0 \\
0 & 0 & 0 & 0 & 0 & \frac{b_{V} \beta_{H} S_{M}^{*}}{N_{H}^{*}} \\
0 & 0 & 0 & 0 & 0 & 0 \\
0 & \frac{b_{V} \beta_{V} S_{V}^{*}}{N_{H}^{*}} & 0 & \frac{b_{V} \beta_{V} S_{V}^{*}}{N_{H}^{*}} & 0 & 0 \\
0 & 0 & 0 & 0 & 0 & 0
\end{array}\right]
$$

$$
\left[\begin{array}{cccccc}
k_{1} & 0 & 0 & 0 & 0 & 0 \\
-\sigma_{F} & k_{2} & 0 & 0 & 0 & 0 \\
0 & 0 & k_{3} & 0 & 0 & 0 \\
0 & 0 & -\sigma_{M} & k_{4} & 0 & 0 \\
0 & 0 & 0 & 0 & k_{5} & 0 \\
0 & 0 & 0 & 0 & -\sigma_{V} & \mu_{V}
\end{array}\right]
$$

where $k_{1}=\sigma_{F}+\mu_{F}, k_{2}=\gamma_{F}+\mu_{F}, k_{3}=\sigma_{M}+\mu_{M}, k_{4}=\gamma_{M}+\mu_{M}, k_{5}=\sigma_{V}+\mu_{V}$. Hence, the reproduction number is given by:

$$
\mathcal{R}_{V}=\rho\left(F_{V} V_{V}^{-1}\right)=\sqrt{\mathcal{R}_{F V}+\mathcal{R}_{M V}},
$$

where $\mathcal{R}_{F V}=\frac{\beta_{H} \sigma_{F} S_{F}^{*} \beta_{V} \sigma_{V} b_{V}^{2} S_{V}^{*}}{k_{1} k_{2} k_{5} \mu_{V}\left(N_{H}^{*}\right)^{2}}$ and $\mathcal{R}_{M V}=\frac{\beta_{H} \sigma_{M} S_{M}^{*} \beta_{V} \sigma_{V} b_{V}^{2} S_{V}^{*}}{k_{3} k_{4} k_{5} \mu_{V}\left(N_{H}^{*}\right)^{2}}$.

Lemma 3. The disease-free equilibrium (DFE) of the Zika virus model (3.1) with mosquito only transmission is locally asymptotically stable $(L A S)$ if $\mathcal{R}_{V}<1$ and unstable if $\mathcal{R}_{V}>1$.

The basic reproduction number $\mathcal{R}_{V}$ is defined as the average number of new infections that result from one infectious individual in a population that is fully susceptible $[4,19,31,63]$. The epidemiological significance of Lemma 3 is that Zika virus can be eliminated from the community if the reproduction number $\left(\mathcal{R}_{V}\right)$ can be brought to (and maintained at) a value less than unity. Figures 2 shows convergence of the solutions of the Zika model (3.1) to the DFE $\left(\mathcal{E}_{V_{1}}\right)$ for the case when $\mathcal{R}_{V}<1$ (in accordance with Lemma 3). 


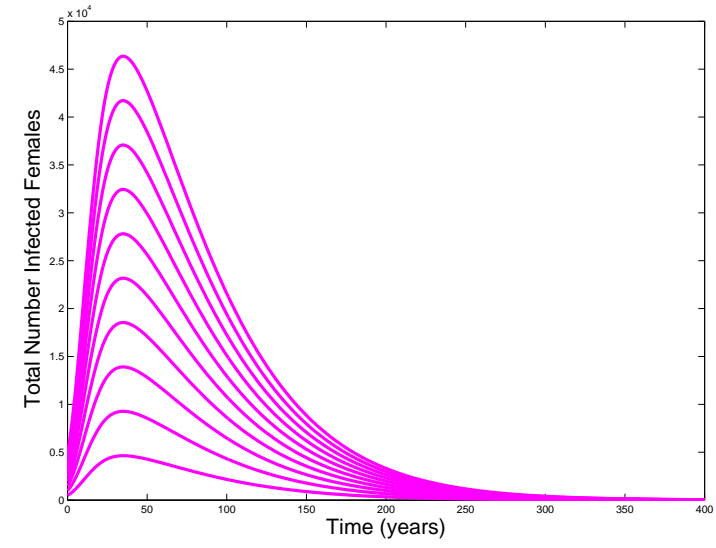

(a)

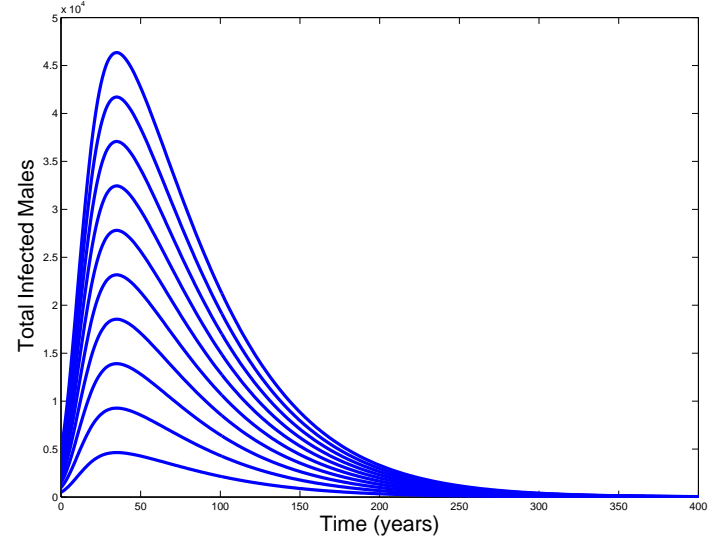

(b)

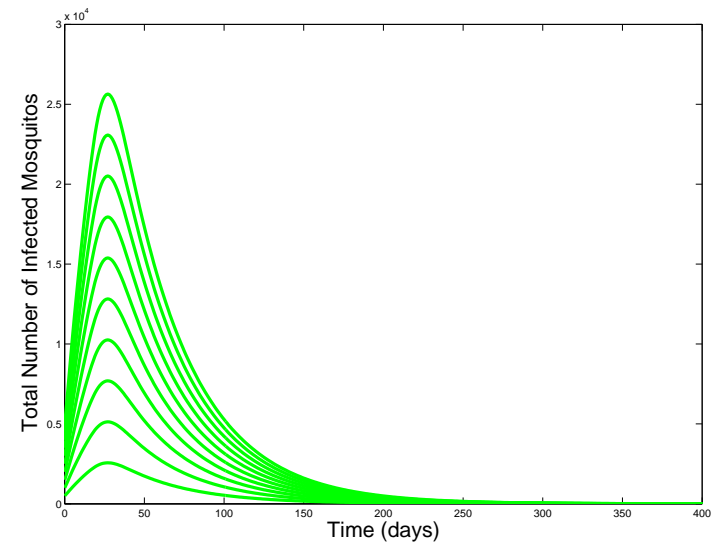

(c)

Figure 2: Simulation of the mosquito transmission route only Zika model (3.1) as a function of time when $\mathcal{R}_{V}<1$. (a) Total number of infectious females (b) Total number of infectious males (c) Total number of infectious mosquitoes. Parameter values used are as given in Table 3.

\subsubsection{Global Asymptotic Stability of the DFE}

Consider the feasible region

$$
\Gamma_{2}=\left\{D \in \Gamma_{M}: S_{F} \leq S_{F}^{*}, S_{M} \leq S_{M}^{*}, S_{V} \leq S_{V}^{*}\right\}
$$

where,

$$
D=\left(S_{F}, E_{F}, I_{F}, R_{F}, S_{M}, E_{M}, I_{M}, R_{M}, S_{V}, E_{V}, I_{V}\right) .
$$

Lemma 4. The region $\Gamma_{2}$ is positively-invariant for the Zika virus model (3.1).

The proof of Lemma 4 is given in Appendix C.

Theorem 1. The DFE $\left(\mathcal{E}_{V_{1}}\right)$ of the Zika virus model (3.1) is globally asymptotically stable (GAS) in $\Gamma_{2}$, whenever $\mathcal{R}_{V} \leq 1$.

The proof of Theorem 1 is given in Appendix D. 


\subsubsection{Existence of Endemic Equilibrium Point (EEP)}

In this section, we will investigate conditions for the existence of endemic equilibria (i.e., equilibria where the infected components of the Zika transmission model (3.1) are non-zero).

Let

$$
\mathcal{E}_{V_{2}}=\left(S_{F}^{* *}, E_{F}^{* *}, I_{F}^{* *}, R_{F}^{* *}, S_{M}^{* *}, E_{M}^{* *}, I_{M}^{* *}, R_{B}^{* *}, S_{V}^{* *}, E_{V}^{* *}, I_{V}^{* *}\right)
$$

be an arbitrary endemic equilibrium of Zika transmission model (3.1), where

$$
\begin{aligned}
S_{F}^{* *} & =\frac{\Pi_{F} N_{H}^{* *}}{\beta_{H} b_{V} I_{V}^{* *}+N_{H}^{* *} \mu_{F}}, & E_{F}^{* *} & =\frac{\beta_{H} b_{V} I_{V}^{* *} \Pi_{F}}{k_{1}\left(b_{V} \beta_{H} I_{V}^{* *}+N_{H}^{* *} \mu_{F}\right)}, \\
I_{F}^{* *} & =\frac{\sigma_{F} \beta_{H} b_{V} I_{V}^{* *} \Pi_{F}}{k_{1} k_{2}\left(b_{V} \beta_{H} I_{V}^{* *}+N_{H}^{* *} \mu_{F}\right)}, & R_{F}^{* *} & =\frac{\gamma_{F} \sigma_{F} b_{V} \beta_{H} I_{V}^{* *} \Pi_{F}}{\mu_{F} k_{1} k_{2}\left(b_{V} \beta_{H} I_{V}^{* *}+N_{H}^{* *} \mu_{F}\right)}, \\
S_{M}^{* *} & =\frac{\Pi_{M} N_{H}^{* *}}{b_{V} \beta_{H} I_{V}^{* *}+N_{H}^{* *} \mu_{M}}, & E_{M}^{* *} & =\frac{b_{V} \beta_{H} I_{V}^{* *} \Pi_{M}}{k_{3}\left(b_{V} \beta_{H} I_{V}^{* *}+N_{H}^{* *} \mu_{M}\right)}, \\
I_{M}^{* *} & =\frac{\sigma_{M} b_{V} \beta_{H} I_{V}^{* *} \Pi_{M}}{k_{3} k_{4}\left(b_{V} \beta_{H} I_{V}^{* *}+N_{H}^{* *} \mu_{M}\right)}, & R_{M}^{* *} & =\frac{\gamma_{M} \sigma_{M} b_{V} \beta_{H} I_{V}^{* *} \Pi_{M}}{\mu_{M} k_{3} k_{4}\left(\beta_{H} b_{V} I_{V}^{* *}+N_{H}^{* *} \mu_{M}\right)}, \\
S_{V}^{* *} & =\frac{\Pi_{V}}{\lambda_{V}^{* *}+\mu_{V}}, & E_{V}^{* *} & =\frac{\lambda_{V}^{* *} \Pi_{V}}{k_{5}\left(\lambda_{V}^{* *}+\mu_{V}\right)}, \\
I_{V}^{* *} & =\frac{\sigma_{V} \lambda_{V}^{*} \Pi_{V}}{k_{5} \mu_{V}\left(\lambda_{V}^{* *}+\mu_{V}\right)}, & &
\end{aligned}
$$

are components of the steady-state solution of the equations of the Zika transmission model (3.1). Also, let

$$
\lambda_{H}^{* *}=\frac{b_{V} \beta_{H} I_{V}^{* *}}{N_{H}^{* *}}, \quad \lambda_{V}^{* *}=\frac{b_{V} \beta_{V}\left(I_{F}^{* *}+I_{M}^{* *}\right)}{N_{H}^{* *}},
$$

be the forces of infection of susceptible humans and mosquitoes at steady-state, respectively. Substituting the expressions for $I_{F}^{* *}$ and $I_{M}^{* *}$, into the equation (3.2) for $\lambda_{V}^{* *}$ and simplifying gives

$$
\lambda_{V}^{* *}=\frac{b_{V}^{2} \beta_{H} \beta_{V} I_{V}^{* *} \mu_{H}^{2}\left[a_{2}\left(I_{V}^{* *}\right)+a_{1}\right]}{k_{1} k_{2} k_{3} k_{4} \Pi_{H}\left(b_{V} \beta_{H} I_{V}^{* *} \mu_{H}+\Pi_{H} \mu_{F}\right)\left(b_{V} \beta_{H} I_{V}^{* *} \mu_{H}+\Pi_{H} \mu_{M}\right)}
$$

where

$a_{2}=b_{V} \beta_{H} \sigma_{M} \Pi_{M} \mu_{H} k_{1} k_{2}+b_{V} \beta_{H} \sigma_{F} \Pi_{F} \mu_{H} k_{3} k_{4}, \quad a_{1}=\sigma_{M} \Pi_{M} \Pi_{H} \mu_{F} k_{1} k_{2}+\sigma_{F} \Pi_{F} \Pi_{H} \mu_{M} k_{3} k_{4}$.

Substituting expression for $I_{V}^{* *}$ into the force of infection $\lambda_{H}^{* *}$ in equation (3.2) gives

$$
\lambda_{H}^{* *}=\frac{\beta_{H} b_{V} \sigma_{V} \lambda_{V}^{* *} \Pi_{V} \mu_{H}}{k_{5} \mu_{V} \Pi_{H}\left(\lambda_{V}^{* *}+\mu_{V}\right)}
$$

and then solving for $\lambda_{V}^{* *}$, gives

$$
\lambda_{V}^{* *}=-\frac{\lambda_{H}^{* *} k_{5} \mu_{V}^{2} \Pi_{H}}{\left(\lambda_{H}^{* *} k_{5} \mu_{V} \Pi_{H}-\beta_{H} b_{V} \sigma_{V} \Pi_{V} \mu_{H}\right)} .
$$


Substituting this result into (3.3), and simplifying, leads to the following quadratic equation

$$
b_{2}\left(\lambda_{H}^{* *}\right)^{2}+b_{1} \lambda_{H}^{* *}+b_{0}\left(1-\mathcal{R}_{V}^{2}\right)=0,
$$

where

$$
\begin{aligned}
b_{2} & =\beta_{V} \mu_{V} \Pi_{H}^{2} k_{5} a_{2}+\beta_{H} \mu_{V}^{2} \Pi_{H}^{3} k_{1} k_{2} k_{3} k_{4} k_{5}, \\
b_{1} & =b_{V} \beta_{V} \beta_{H} \Pi_{H} \mu_{H}\left(\mu_{V} k_{5} a_{1}-\sigma_{V} \Pi_{V} a_{2}\right)+\beta_{H} \Pi_{H}^{3} \mu_{V}^{2} k_{1} k_{2} k_{3} k_{4} k_{5}\left(\mu_{F}+\mu_{M}\right), \\
b_{0} & =\Pi_{H}^{3} \beta_{H} \mu_{F} \mu_{M} \mu_{V}^{2} k_{1} k_{2} k_{3} k_{4} k_{5} .
\end{aligned}
$$

From (3.4), it can easily be seen that $b_{2}>0$. Further, $b_{0}\left(1-\mathcal{R}_{V}^{2}\right)<0$ whenever $\mathcal{R}_{V}^{2}>1$. Thus, the number of possible positive real roots the polynomial (3.4) can have depends on the signs of $b_{1}$. This can be analyzed using the Descartes Rule of Signs on the quadratic polynomial $g(x)=c_{2} x^{2}+c_{1} x+c_{0}$, given in (3.4) (with $\left.x=\lambda_{H}^{* *}, c_{2}=b_{2}, c_{1}=b_{1}, c_{0}=b_{0}\left(1-\mathcal{R}_{V}^{2}\right)\right)$. The various possibilities for the roots of $g(x)$ are tabulated in Table 2.

\begin{tabular}{|l|c|c|l|l|c|c|}
\hline \hline Cases & $c_{2}$ & $c_{1}$ & $c_{0}$ & $\mathcal{R}_{V}$ & Number of sign changes & $\begin{array}{c}\text { No of possible positive real roots } \\
\text { (disease-free/endemic equilibrium) }\end{array}$ \\
\hline 1 & + & + & - & $\mathcal{R}_{V}>1$ & 1 & 1 \\
\hline 2 & + & - & - & $\mathcal{R}_{V}>1$ & 1 & 1 \\
\hline
\end{tabular}

Table 2: Number of Possible Positive Real Roots of $g(x)$ for $\mathcal{R}_{V}>1$.

The following results (Theorem 2) follow from the various possible combinations for the roots of $g(x)$, in Table 2 .

Theorem 2. The Zika transmission model (3.1) has a unique endemic equilibrium if $\mathcal{R}_{V}>1$.

Numerical simulations of the Zika transmission model (3.1), depicted in Figure 3, show convergence to an endemic equilibrium when $\mathcal{R}_{V}>1$ (suggesting that the unique EEP of the Zika transmission model (3.1) is asymptotically stable when it exists).

\subsubsection{Local stability of the endemic equilibrium (EE)}

In this Section we will consider the stability of the endemic equilibrium of model (3.1). Let

$$
\mathcal{E}_{V_{2}}=\left(S_{F}^{* *}, E_{F}^{* *}, I_{F}^{* *}, R_{F}^{* *}, S_{M}^{* *}, E_{M}^{* *}, I_{M}^{* *}, R_{M}^{* *}, S_{V}^{* *}, E_{V}^{* *}, I_{V}^{* *}\right)
$$

be the unique endemic equilibrium of the Zika model (3.1).

Lemma 5. The unique endemic equilibrium $\left(\mathcal{E}_{V_{2}}\right)$ of model (3.1) is locally asymptotically stable.

The proof of Lemma 5 is given in Appendix E. Numerical simulations of the Zika model (3.1), depicted in Figure 3, show convergence to a unique endemic equilibrium when $\mathcal{R}_{V}>1$ (suggesting that the unique EEP of the Zika model (3.1) is asymptotically stable when it exists). 


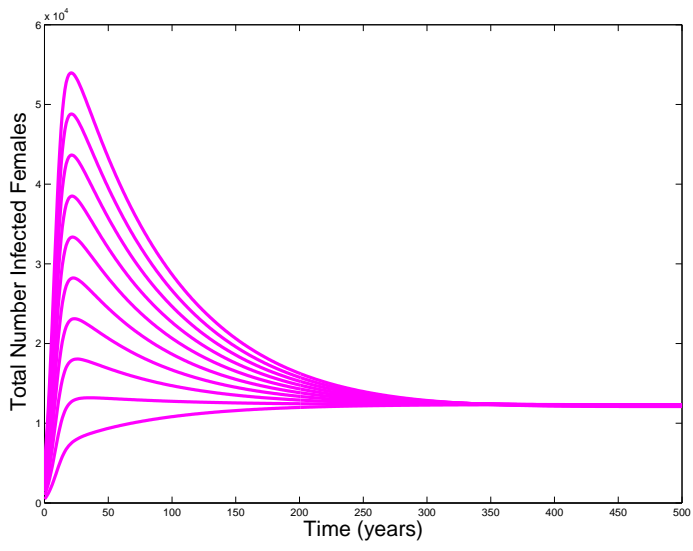

(a)

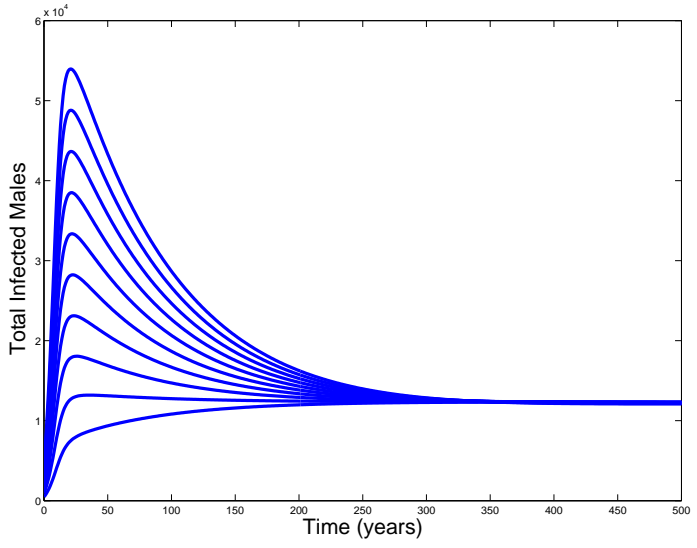

(b)

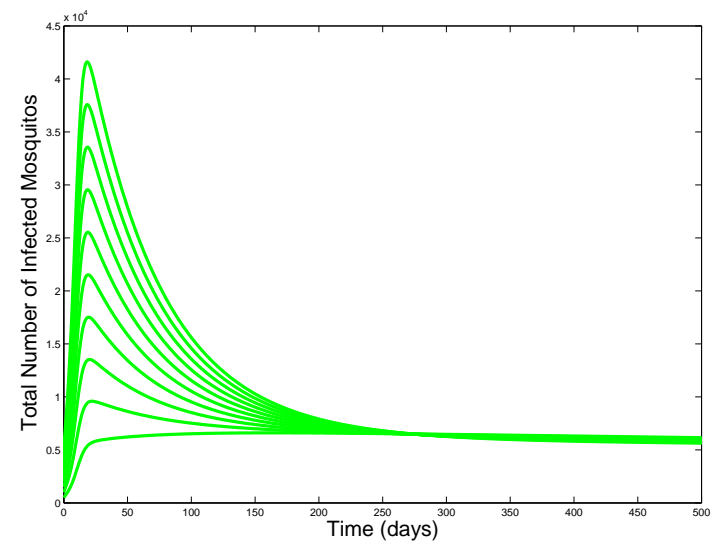

(c)

Figure 3: Simulation of the mosquito transmission route only Zika model (3.1) as a function of time when $\mathcal{R}_{V}>1$. (a) Total number of infectious females (b) Total number of infectious males (c) Total number of infectious mosquitoes. Parameter values used are as given in Table 3

\subsection{Sexual transmission route only model}

The sexual transmission route only model is obtained by assuming that Zika virus is only trans- 
to the following

$$
\begin{aligned}
\frac{d S_{F}}{d t} & =\Pi_{F}-\frac{C_{M_{1}} \beta_{M} I_{M} S_{F}}{N_{F}}-\mu_{F} S_{F} \\
\frac{d E_{F}}{d t} & =\frac{C_{M_{1}} \beta_{M} I_{M} S_{F}}{N_{F}}-\left(\sigma_{F}+\mu_{F}\right) E_{F} \\
\frac{d I_{F}}{d t} & =\sigma_{F} E_{F}-\left(\gamma_{F}+\mu_{F}\right) I_{F} \\
\frac{d R_{F}}{d t} & =\gamma_{F} I_{F}-\mu_{F} R_{F} \\
\frac{d S_{M}}{d t} & =\Pi_{M}-\frac{C_{F} \beta_{F} I_{F} S_{M}}{N_{M}}-\frac{C_{M_{2}} \beta_{M} I_{M} S_{M}}{N_{M}}-\mu_{M} S_{M} \\
\frac{d E_{M}}{d t} & =\frac{C_{F} \beta_{F} I_{F} S_{M}}{N_{M}}+\frac{C_{M_{2}} \beta_{M} I_{M} S_{M}}{N_{M}}-\left(\sigma_{M}+\mu_{M}\right) E_{M} \\
\frac{d I_{M}}{d t} & =\sigma_{M} E_{M}-\left(\gamma_{M}+\mu_{M}\right) I_{M} \\
\frac{d R_{M}}{d t} & =\gamma_{M} I_{M}-\mu_{M} R_{M} .
\end{aligned}
$$

For this model (3.5), it can be shown (using the approaches in Section 2.1) that the feasible region,

$$
\Gamma_{S} \subset \mathbb{R}_{+}^{8}
$$

Thus, the reproduction number for the model (3.5) is given by

$$
\mathcal{R}_{S}=\rho\left(F_{S} V_{S}^{-1}\right)=\frac{1}{2} \frac{C_{M_{2}} \beta_{M} \sigma_{M}}{k_{3} k_{4}}+\frac{1}{2} \sqrt{\frac{\beta_{M} \sigma_{M}}{k_{3} k_{4}}\left[\frac{4 C_{F} \beta_{F} \sigma_{F} C_{M_{1}}}{k_{1} k_{2}}+\frac{C_{M_{2}}^{2} \beta_{M} \sigma_{M}}{k_{3} k_{4}}\right]} .
$$


Lemma 6. The disease-free equilibrium (DFE) of the Zika virus model (3.5) with sexual transmission route is locally asymptotically stable (LAS) if $\mathcal{R}_{S}<1$ and unstable if $\mathcal{R}_{S}>1$.

The epidemiological significance of Lemma 6 is that, Zika virus will be eliminated from the community if the reproduction number $\left(R_{S}\right)$ can be brought to (and maintained at) a value less than unity.

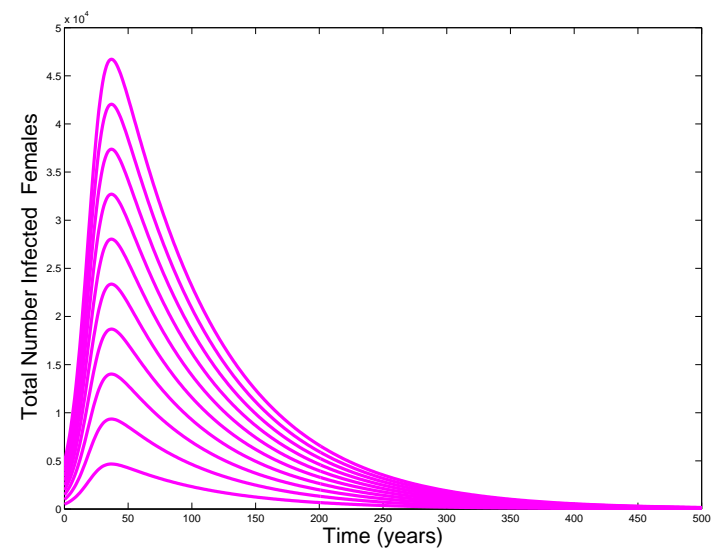

(a)

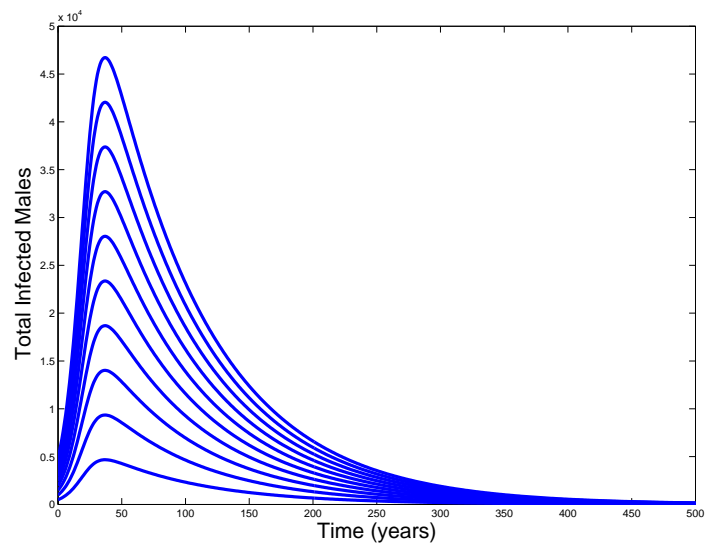

(b)

Figure 4: Simulation of the sexual transmission route only Zika model (3.5) as a function of time when $\mathcal{R}_{S}<1$. (a) Total number of infectious females (b) Total number of infectious males. Parameter values used are as given in Table 3.

\subsubsection{Global stability of the DFE}

Theorem 3. The DFE of the sexual transmission route only model (3.5), given by $\mathcal{E}_{S_{1}}$, is GAS in $\Gamma$ whenever $\mathcal{R}_{S}<1$.

The proof of Theorem 3 is given in Appendix F.

The above result shows that, with only the sexual transmission route, Zika will be eliminated from the community if the threshold quantity $\mathcal{R}_{S}$ can be brought to a value less than unity.

\subsubsection{Existence of Endemic Equilibrium Point (EEP)}

In this section, we will investigate conditions for the existence of endemic equilibria of the Zika transmission model (3.5).

Let

$$
\mathcal{E}_{S_{2}}=\left(S_{F}^{* *}, E_{F}^{* *}, I_{F}^{* *}, R_{F}^{* *}, S_{M}^{* *}, E_{M}^{* *}, I_{M}^{* *}, R_{B}^{* *}\right)
$$

be an arbitrary endemic equilibrium of Zika transmission model (3.5). We will study the existence of this equilibrium $\left(\mathcal{E}_{S_{2}}\right)$ under two scenarios: when there are only heterosexual couples present, and when there are only homosexual couples present.

Let $\tilde{\mathcal{E}}_{S_{21}}=\left(S_{F}^{* *}, E_{F}^{* *}, I_{F}^{* *}, R_{F}^{* *}, S_{M}^{* *}, E_{M}^{* *}, I_{M}^{* *}, R_{M}^{* *}\right)$ be an arbitrary equilibrium of the Zika model (3.5) with heterosexual only couples, where 


$$
\begin{aligned}
S_{F}^{* *} & =\frac{\Pi_{F}}{\lambda_{F M}^{* *}+\mu_{F}}, & E_{F}^{* *} & =\frac{\Pi_{F} \lambda_{F M}^{* *}}{k_{1}\left(\lambda_{F M}^{* *}+\mu_{F}\right)}, \\
I_{F}^{* *} & =\frac{\sigma_{F} \Pi_{F} \lambda_{F M}^{* *}}{k_{1} k_{2}\left(\lambda_{F M}^{* *}+\mu_{F}\right)}, & R_{F}^{* *} & =\frac{\gamma_{F} \sigma_{F} \Pi_{F} \lambda_{F M}^{* *}}{k_{1} k_{2} \mu_{F}\left(\lambda_{F M}^{* *}+\mu_{F}\right)}, \\
S_{M}^{* *} & =\frac{\Pi_{M}}{\lambda_{M F}^{* *}+\mu_{M}}, & E_{M}^{* *} & =\frac{\Pi_{M} \lambda_{M F}^{* *}}{k_{3}\left(\lambda_{M F}^{* *}+\mu_{M}\right)}, \\
I_{M}^{* *} & =\frac{\sigma_{M} \Pi_{M} \lambda_{M F}^{* *}}{k_{3} k_{4}\left(\lambda_{M F}^{* *}+\mu_{M}\right)}, & R_{M}^{* *} & =\frac{\gamma_{M} \sigma_{M} \Pi_{M} \lambda_{M F}^{* *}}{k_{3} k_{4} \mu_{M}\left(\lambda_{M F}^{* *}+\mu_{M}\right)},
\end{aligned}
$$

are components of the steady-state solution of the equations of the Zika transmission model (3.5) with heterosexual only couples. Also, let

$$
\lambda_{F M}^{* *}=\frac{C_{M_{1}} \beta_{M} I_{M}^{* *}}{N_{F}^{* *}}, \quad \lambda_{M F}^{* *}=\frac{C_{F} \beta_{F} I_{F}^{* *}}{N_{M}^{* *}}
$$

be the forces of infection of susceptible heterosexual females and males at steady-state, respectively. Substituting the expressions for $I_{F}^{* *}$, into equation (3.6) for $\lambda_{M F}^{* *}$ and simplifying gives

$$
\lambda_{M F}^{* *}=\frac{C_{F} \beta_{F} \sigma_{F} \Pi_{F} \mu_{M} \lambda_{F M}^{* *}}{k_{1} k_{2} \Pi_{M}\left(\lambda_{F M}^{* *}+\mu_{F}\right)}
$$

Substituting the expression for $I_{M}^{* *}$ into the force of infection $\lambda_{F M}^{* *}$ in equation (3.6) gives

$$
\lambda_{F M}^{* *}=\frac{C_{M_{1}} \beta_{M} \sigma_{M} \Pi_{M} \mu_{F} \lambda_{M F}^{* *}}{k_{3} k_{4} \Pi_{F}\left(\lambda_{M F}^{* *}+\mu_{M}\right)}
$$

and then solving (3.8) for $\lambda_{M F}^{* *}$, gives

$$
\lambda_{M F}^{* *}=-\frac{\lambda_{F M}^{* *} k_{3} k_{4} \Pi_{F} \mu_{M}}{\lambda_{F M}^{* *} k_{3} k_{4} \Pi_{F}-C_{M_{1}} \beta_{M} \sigma_{M} \Pi_{M} \mu_{F}} .
$$

Substituting this result into (3.7), and simplifying, leads to the following linear equation

$$
\begin{gathered}
\Pi_{F} \mu_{M}\left(C_{F} \beta_{F} \sigma_{F} k_{4} k_{3} \Pi_{F}+k_{4} k_{3} k_{2} k_{1} \Pi_{M}\right) \lambda_{F M}^{* *}+\Pi_{F} \mu_{M} k_{4} k_{3} k_{2} k_{1} \Pi_{M} \mu_{F}\left(1-\tilde{\mathcal{R}}_{S}^{2}\right)=0, \\
\lambda_{F M}^{* *}=\frac{\Pi_{F} \mu_{M} k_{4} k_{3} k_{2} k_{1} \Pi_{M} \mu_{F}\left(\tilde{\mathcal{R}}_{S}^{2}-1\right)}{\Pi_{F} \mu_{M}\left(C_{F} \beta_{F} \sigma_{F} k_{4} k_{3} \Pi_{F}+k_{4} k_{3} k_{2} k_{1} \Pi_{M}\right)} .
\end{gathered}
$$

The quantity $\tilde{\mathcal{R}}_{S}^{2}=\frac{C_{F} \beta_{F} \sigma_{F} C_{M_{1}} \beta_{M} \sigma_{M}}{k_{1} k_{2} k_{3} k_{4}}$, is the reproduction number when the community consists purely of heterosexual couples. Thus,

$$
\lambda_{F M}^{* *}>0, \text { provided } \tilde{\mathcal{R}}_{S}^{2}>1 .
$$

Hence, the polynomial (3.9) has only one root. This result is summarized in the following Theorem 4 below. 
Theorem 4. The Zika transmission model (3.5) with purely heterosexual couples has a unique endemic equilibrium if $\tilde{\mathcal{R}}_{S}>1$.

Notice, however, that if Zika sexual transmission only occurs from males to females, and not in the other direction, then $\beta_{F}=0$ and $\tilde{\mathcal{R}}_{S}^{2}=0$. Thus, as expected, sexual transmission among purely heterosexual couples must be bidirectional in order to support continued Zika transmission in the absence of other modes of spread.

Let $\tilde{\mathcal{E}}_{S_{22}}=\left(S_{F}^{* *}, 0,0,0, S_{M}^{* *}, E_{M}^{* *}, I_{M}^{* *}, R_{M}^{* *}\right)$ be an arbitrary equilibrium of the model with only sexual transmission (3.5) and only homosexual male couples present. In this case

$$
\begin{aligned}
S_{F}^{* *} & =\frac{\Pi_{F}}{\mu_{F}}, & S_{M}^{* *} & =\frac{\Pi_{M}}{\lambda_{M}^{* *}+\mu_{M}}, \\
E_{M}^{* *} & =\frac{\Pi_{M} \lambda_{M}^{* *}}{k_{3}\left(\lambda_{M}^{* *}+\mu_{M}\right)}, & I_{M}^{* *} & =\frac{\sigma_{M} \Pi_{M} \lambda_{M}^{* *}}{k_{3} k_{4}\left(\lambda_{M}^{* *}+\mu_{M}\right)}, \\
R_{M}^{* *} & =\frac{\gamma_{M} \sigma_{M} \Pi_{M} \lambda_{M}}{k_{3} k_{4} \mu_{M}\left(\lambda_{M}^{* *}+\mu_{M}\right)}, & \lambda_{M}^{* *} & =\frac{C_{M 2} \beta_{M} I_{M}^{* *}}{N_{M}^{* *}} .
\end{aligned}
$$

Furthermore, the quantity $\lambda_{M}^{* *}$ is the force of infection at the endemic equilibrium steady states for homosexual male couples. Using the approach in the proof of uniqueness of the equilibrium $\tilde{\mathcal{E}}_{S_{21}}$, we have

$$
k_{3} k_{4} \lambda_{M}^{* *}+k_{3} k_{4} \mu_{M}\left(1-\mathcal{R}_{M S}\right)=0,
$$

where $\mathcal{R}_{M S}=\frac{C_{M 2} \beta_{M} \sigma_{M}}{k_{3} k_{4}}$, is the reproduction number for homosexual male only couples. Thus,

$$
\lambda_{M}^{* *}>0, \quad \text { provided } \mathcal{R}_{M S}>1 .
$$

Hence, $\tilde{\mathcal{E}}_{S_{22}}$ is a unique equilibrium for model (3.5) when only homosexual males transmit the virus.

Theorem 5. The Zika transmission model (3.5) with only homosexual male couples has a unique endemic equilibrium if $\mathcal{R}_{M S}>1$.

Notice that, in contrast to our scenario with purely heterosexual transmission, the reproduction number for our scenario with purely homosexual transmission only depends on a single transmission probability, reflecting the higher sexual symmetry of male-to-male pairings. Notably, however, this assumes that homosexual men are predominantly versatile (i.e., engage in both insertive and receptive anal intercourse), which appears to be the case for a large portion of the population, though not the population as a whole [37].

Numerical simulations of the Zika virus transmission model (3.5), depicted in Figure 5, show convergence to an endemic equilibrium when $\mathcal{R}_{S}>1$ (suggesting that the unique EEP of the Zika transmission model (3.5) is asymptotically stable when it exists). 


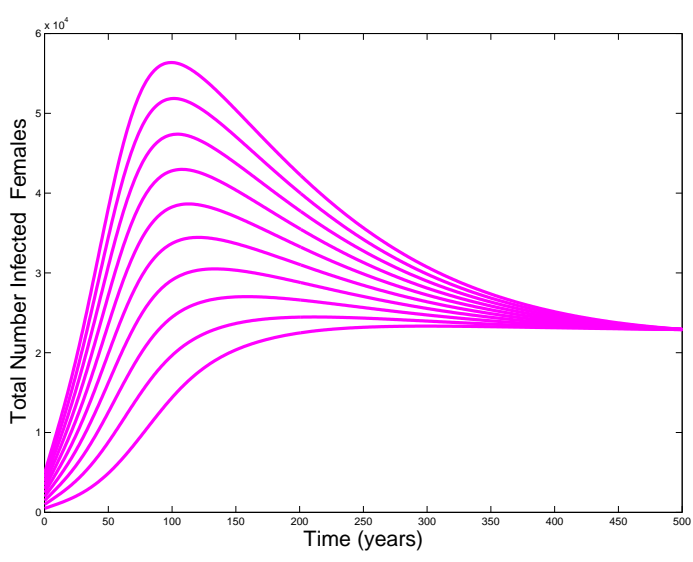

(a)

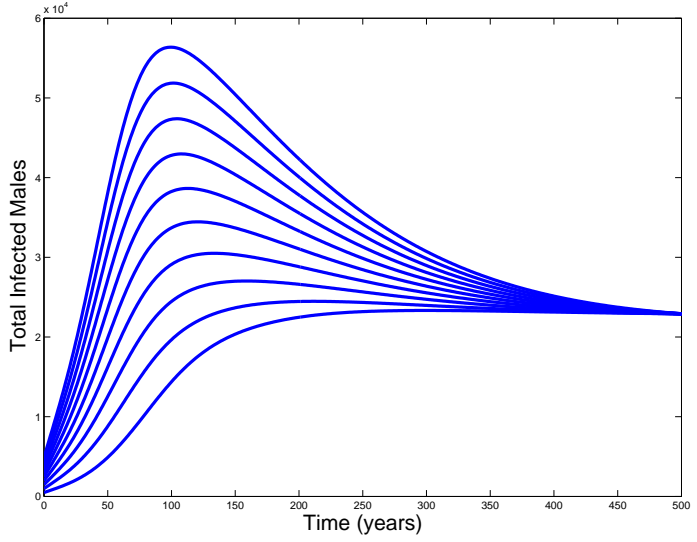

(b)

Figure 5: Simulation of the sexual transmission route only Zika model (3.5) as a function of time when $\tilde{\mathcal{R}}_{S}>1$. (a) Total number of infectious females (b) Total number of infectious males. Parameter values used are as given in Table 3

\subsubsection{Local Stability of the Endemic Equilibrium}

As above, we will study the stability of the endemic equilibrium for the sexual transmission route only model (3.5) under scenarios where only heterosexuals couples are present and under scenarios where only homosexual couples are present.

Let $\tilde{\mathcal{E}}_{S_{21}}=\left(S_{F}^{* *}, E_{F}^{* *}, I_{F}^{* *}, R_{F}^{* *}, S_{M}^{* *}, E_{M}^{* *}, I_{M}^{* *}, R_{M}^{* *}\right)$ be the unique equilibrium of the Zika model (3.5) with only heterosexual couples.

Lemma 7. The unique endemic equilibrium $\left(\mathcal{E}_{S_{21}}\right)$ of the sexual transmission route only Zika model (3.5) with only heterosexual couples is locally asymptotically stable.

The proof of Lemma 7 is given in Appendix G.

Let $\tilde{\mathcal{E}}_{S_{22}}=\left(S_{F}^{* *}, 0,0,0, S_{M}^{* *}, E_{M}^{* *}, I_{M}^{* *}, R_{M}^{* *}\right)$ be the unique equilibrium of the sexual transmission route only Zika model (3.5) with only homosexual male couples.

Lemma 8. The unique endemic equilibrium $\left(\tilde{\mathcal{E}}_{S_{22}}\right)$ of the sexual transmission route Zika model (3.5) with only homosexual male couples is locally asymptotically stable.

The proof of Lemma 8 is given in Appendix $\mathrm{H}$.

\subsection{Analysis of the Full Model}

\subsubsection{Local stability of disease-free equilibrium}

The disease-free equilibrium of the full Zika model (2.5) is given as

$$
\begin{aligned}
\mathcal{E}_{F_{1}} & =\left(S_{F}^{*}, E_{F}^{*}, I_{F}^{*}, R_{F}^{*}, S_{M}^{*}, E_{M}^{*}, I_{M}^{*}, R_{B}^{*}, S_{V}^{*}, E_{V}^{*}, I_{V}^{*}\right) \\
& =\left(\frac{\Pi_{F}}{\mu_{F}}, 0,0,0, \frac{\Pi_{M}}{\mu_{M}}, 0,0,0, \frac{\Pi_{V}}{\mu_{V}}, 0,0\right) .
\end{aligned}
$$


The local asymptotic stability of $\mathcal{E}_{F_{1}}$ can be established using the next generation matrix method on the system (2.5). Taking the infected compartments $\left(E_{F}^{*}, I_{F}^{*}, E_{M}^{*}, I_{M}^{*}, E_{V}^{*}, I_{V}^{*}\right)$ at the DFE and using the notation in [63], the Jacobian matrices $F_{F}$ and $V_{F}$ for the new infection terms and the remaining transfer terms are respectively given by,

$$
F_{F}=\left[\begin{array}{cccccc}
0 & 0 & 0 & C_{M_{1}} \beta_{M} & 0 & \frac{b_{V} \beta_{H} S_{F}^{*}}{N_{H}^{*}} \\
0 & 0 & 0 & 0 & 0 & 0 \\
0 & C_{F} \beta_{F} & 0 & C_{M_{2}} \beta_{M} & 0 & \frac{b_{V} \beta_{H} S_{M}^{*}}{N_{H}^{*}} \\
0 & 0 & 0 & 0 & 0 & 0 \\
0 & \frac{b_{V} \beta_{V} S_{V}^{*}}{N_{H}^{*}} & 0 & \frac{b_{V} \beta_{V} S_{V}^{*}}{N_{H}^{*}} & 0 & 0 \\
0 & 0 & 0 & 0 & 0 & 0
\end{array}\right], \quad V_{F}=\left[\begin{array}{cccccc}
k_{1} & 0 & 0 & 0 & 0 & 0 \\
-\sigma_{F} & k_{2} & 0 & 0 & 0 & 0 \\
0 & 0 & k_{3} & 0 & 0 & 0 \\
0 & 0 & -\sigma_{M} & k_{4} & 0 & 0 \\
0 & 0 & 0 & 0 & k_{5} & 0 \\
0 & 0 & 0 & 0 & -\sigma_{V} & \mu_{V}
\end{array}\right] .
$$

The reproduction number is determined by the spectral radius of the following matrix

$$
\mathcal{R}_{F}=\rho\left(F_{F} V_{F}^{-1}\right) .
$$

Lemma 9. The disease-free equilibrium (DFE) of the Zika virus model (2.5) is locally asymptotically stable $(L A S)$ if $\mathcal{R}_{F}<1$ and unstable if $\mathcal{R}_{F}>1$.

\subsection{Backward Bifurcation Analysis: Special Case}

Zika associated deaths in infected individuals are rare $[15,23]$ since symptoms of the disease are generally mild. Despite this, a number of deaths have been associated with infection complications in Puerto Rico [20], Venezuela [35], Colombia [55], and more recently in the US [9]. Specifically, in Colombia, fatally occurred from 48 hours to 12 days after the onset of symptoms; all the patients were presented with anaemia before the occurrence of fatality and they all died within 24 hours of admission into the hospital [55]. The patient in US developed septic shock with multiple organ failure; the patient had viremic level that was approximately 100,000 times higher than the average level reported in persons infected with Zika virus [9]. It should be noted that the reported deaths were not in children with microcephaly neither were these in adults who were known to have Guillan-Barre syndrome $[9,20,35,55]$.

Thus, to explore the effect that disease induced death might have on model predictions, we introduced into the symptomatic human compartments disease-induced mortality parameters $\left(\delta_{F}, \delta_{M}\right)$. Thus, the symptomatic human compartments of the Zika model (2.5) can be written as follows:

$$
\begin{aligned}
\frac{d I_{F}}{d t} & =\sigma_{F} E_{F}-\left(\gamma_{F}+\mu_{F}+\delta_{F}\right) I_{F} \\
\frac{d I_{M}}{d t} & =\sigma_{M} E_{M}-\left(\gamma_{M}+\mu_{M}+\delta_{M}\right) I_{M}
\end{aligned}
$$

It can be shown that the reproduction number for the Zika model with disease induced mortality is given by

$$
\tilde{\mathcal{R}}_{F}=\left.\mathcal{R}_{F}\right|_{\delta_{F}, \delta_{M} \neq 0}=\rho\left(F_{F} V_{F}^{-1}\right),
$$


where, $k_{1}=\sigma_{F}+\mu_{F}, k_{2}=\gamma_{F}+\mu_{F}+\delta_{F}, k_{3}=\sigma_{M}+\mu_{M}, k_{4}=\gamma_{M}+\mu_{M}+\delta_{M}, k_{5}=\sigma_{V}+\mu_{V}$.

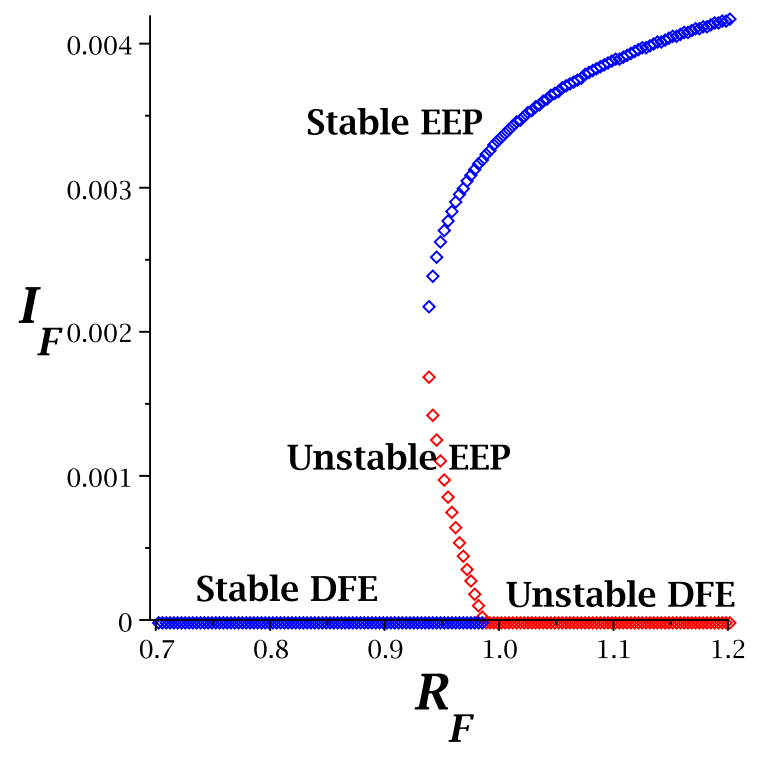

(a)

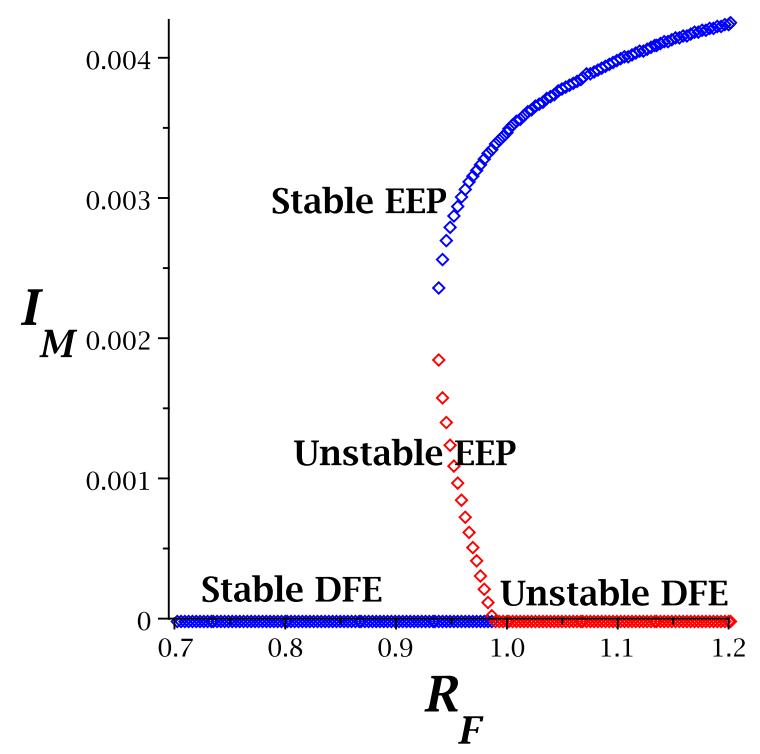

(b)

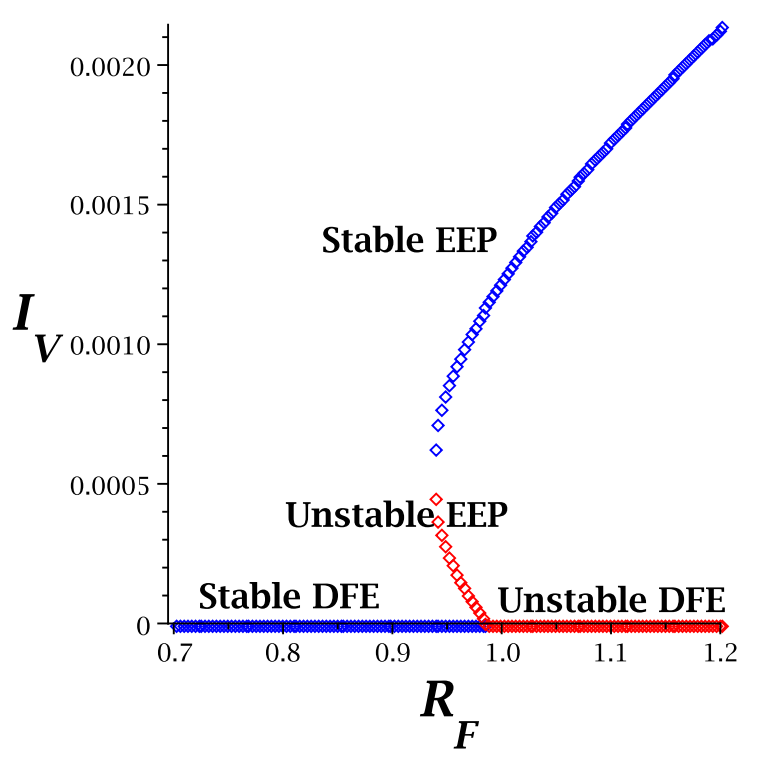

(c)

Figure 6: Backward bifurcation plot of the Zika model (2.5) with the symptomatic human compartments given in (3.10). (a). Symptomatic females; (b). Symptomatic males. (c). Infected mosquitoes. Parameter values used are as given in Table 3.

Models of disease transmission typically undergo a simple transcritical bifurcation (exchange of stability from the DFE to an endemic equilibrium) at $\mathcal{R}_{0}=1$. However, backward bifurcation, where a stable DFE co-exists with a stable endemic equilibrium when the reproduction number, $\tilde{\mathcal{R}}_{F}$, is less than unity $[1,2,7,11,26]$, is also possible. The epidemiological implication of backward bifurcation is that the effective control (or elimination) of Zika virus in the system is dependent 


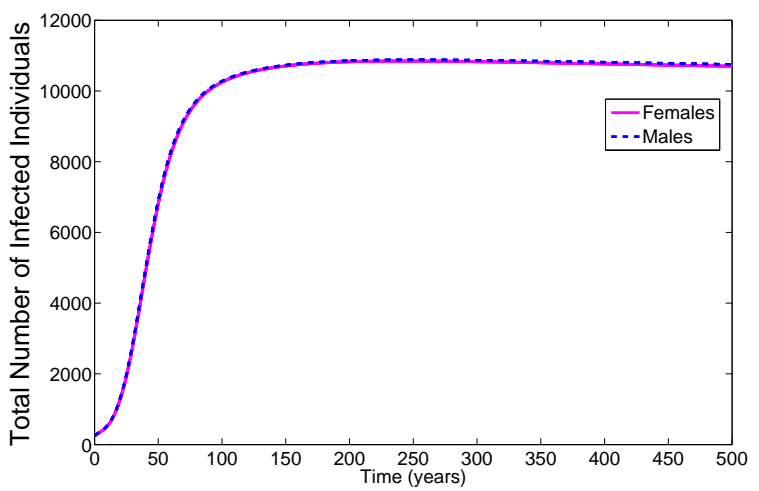

(a)

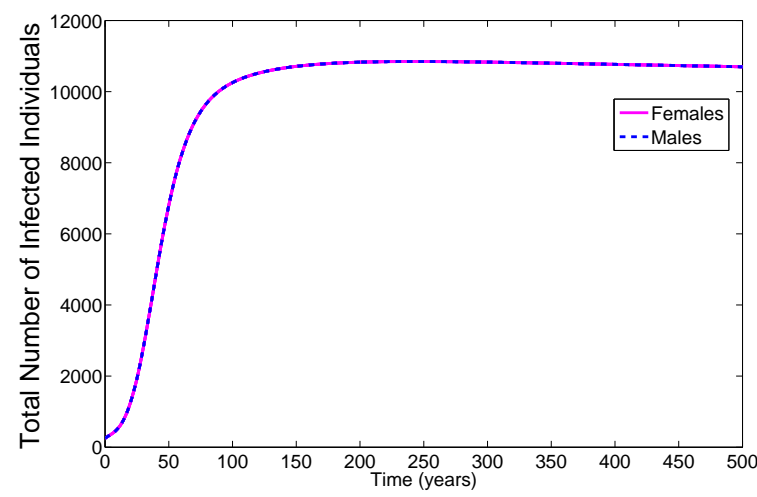

(b)

Figure 7: Simulation of the Zika model (2.5) as a function of time with different sexual contact rates. Parameter values used are as given in Table 3.(a). Setting the sexual contact rate $C_{F}=C_{M_{1}}=C_{M_{2}}=2$. (b). Setting the sexual contact rate $C_{F}=0, C_{M_{1}}=C_{M_{2}}=2$. 


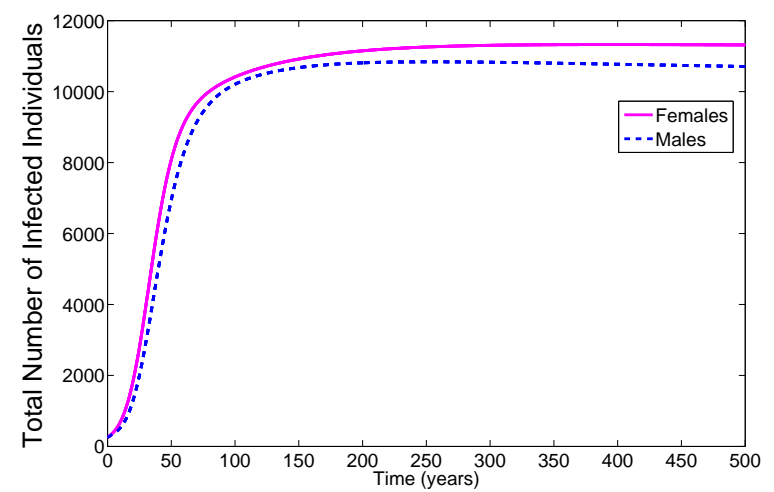

(a)

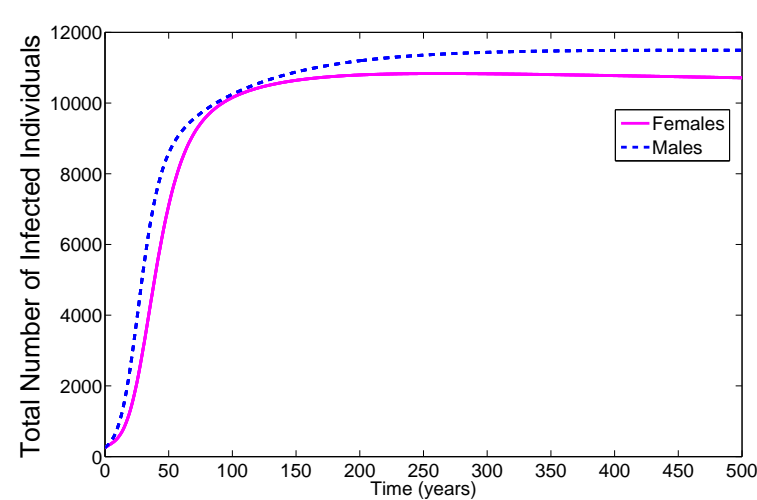

(b)

Figure 8: Simulation of the Zika model (2.5) as a function of time with different sexual contact rates. Parameter values used are as given in Table 3.(a). Setting the sexual contact rate $C_{F}=0, C_{M_{1}}=$ 50, $C_{M_{2}}=2$. (b). Setting the sexual contact rate $C_{F}=0, C_{M_{1}}=2, C_{M_{2}}=80$.

\begin{tabular}{lll}
\hline \hline Parameter & Values & References \\
\hline$\Pi_{H}$ & $\frac{1}{15 \times 365}$ & {$[65]$} \\
$C_{F}, C_{M_{1}}, C_{M_{2}}$ & $2 / 365$ & Assumed \\
$\beta_{H}$ & 0.33 & {$[24,25,38,52,62]$} \\
$\sigma_{F}$ & $\frac{1}{7.5}$ & {$[6]$} \\
$\gamma_{F}$ & $\frac{1}{8.5} 1$ & {$[6]$} \\
$\mu_{F}$ & $\frac{70 \times 365}{705}$ & {$[38]$} \\
$\beta_{F}, \beta_{M}$ & $1-(1-0.001)^{60}$ & {$[28]$} \\
$\sigma_{M}$ & $\frac{1}{7.5}$ & {$[6]$} \\
$\gamma_{M}$ & $\frac{1}{8.5}$ & {$[6]$} \\
$\mu_{M}$ & $\frac{1}{18.6 \times \times 365}$ & {$[66]$} \\
$\delta_{M}$ & $\frac{38}{3174 \times 365}$ & {$[65]$} \\
$\Pi_{V}$ & 500 & {$[6]$} \\
$\beta_{V}$ & 0.33 & {$[38,46,50]$} \\
$b_{V}$ & 0.5 & {$[53,60,38]$} \\
$\sigma_{V}$ & $\frac{1}{3.5}$ & {$[22,25,44,56]$} \\
$\mu_{V}$ & $\frac{1}{21}$ & {$[57,60,61]$} \\
& & \\
\hline \hline
\end{tabular}

Table 3: Parameter values of model (2.5)

Next, we assume that men cannot contract the virus from infected females, additionally, we also 
assume males in the population have multiple female sexual partners while the homosexual males maintain a monogamous relationship, we observed in Figure 8(a) that this risky behavior leads to more infected females than males with $2.37 \%$ difference between females and males, see also Table 4. When the men maintain a monogamous relationship with females in the population but have multiple sexual partners as homosexual males, we observed that this risky behavior leads to more infected males compared to females, this resulted in about $3.04 \%$ difference between the sexes (see Table 4).

\begin{tabular}{lll}
\hline \hline Sexual contact rates & Females & Males \\
\hline$C_{F}=C_{M_{1}}=C_{M_{2}}=2$ & $4.894213 \times 10^{6}$ & $4.912264 \times 10^{6}$ \\
$C_{F}=0, C_{M_{1}}=C_{M_{2}}=2$ & $4.894010 \times 10^{6}$ & $4.894010 \times 10^{6}$ \\
$C_{F}=0, C_{M_{1}}=50, C_{M_{2}}=2$ & $5.134227 \times 10^{6}$ & $4.895659 \times 10^{6}$ \\
$C_{F}=0, C_{M_{1}}=2, C_{M_{2}}=80$ & $4.897354 \times 10^{6}$ & $5.204081 \times 10^{6}$ \\
\hline \hline
\end{tabular}

Table 4: Simulation results of the Zika model (2.5) for the cumulative number of infected cases over the simulation period from 0 to 500 days with different sexual contact rates. Parameter values used are as given in Table 3 .

We further explore the scenario with no male-to-female transmission and compare this with the transmission in the absence of mosquitoes. We observed in Table 5 more infection in both female and male populations when there are no male-to-female transmission and compare to the transmission in the absence of mosquitoes. This result points to the importance of the sexual route transmission.

\begin{tabular}{lll}
\hline \hline & Females & Males \\
\hline Sexual-only transmission & $4.874287 \times 10^{6}$ & $4.874287 \times 10^{6}$ \\
Mosquitoes-only transmission & $8.253976 \times 10^{4}$ & $1.137854 \times 10^{5}$ \\
\hline \hline
\end{tabular}

Table 5: Simulation results of the Zika model (2.5) for the total number of infected cases with no sexual contact rate and in the absences of mosquitoes. Parameter values used are as given in Table 3.

\section{Discussion and Conclusion}

In this paper, we develop a new deterministic model to study the transmission dynamics of Zika virus. Our model incorporates both vector-borne and sexual transmission routes. Additionally, it considers both heterosexual and homosexual transmission. Sexual transmission of Zika is interesting for several reasons. First, Zika is the only known arbovirus to exhibit a sexual mode of spread in the human population. Second, unlike most purely venereal diseases, Zika virus appears to exhibit unidirectional transmission. In particular, Zika appears to transmit from males to females, and from males to males, but not from females to males. Although data is still limited, if the trend continues to hold, then of Zika virus cannot persist in a heterosexual population as a purely venereal disease.

By contrast to a heterosexual population, however, Zika sexual transmission may be self-sustaining in a homosexual population. This is because versatility, which is relatively common [28], allows spread to all partners. Consequently, in the absence of mosquitoes and, assuming no bisexuality, Zika would be restricted to homosexual populations. Bisexual males, however, can enable spillover from the homosexual population to the heterosexual population, although this will primarily be to 
females. Likewise, vector transmission can also allow disease spread between the sub-populations, enabling long-term disease incidence, including among heterosexual men. Interestingly, however, even within the context of vector-borne transmission the biased directionality of spread between females and males during sexual contact, creates a situation where different sexual modes can result in higher risks to different sub-populations. If, for example, heterosexual couples are more promiscuous than homosexual couples, then disease incidence rises in the female population, creating a large female:male ratio of Zika infections. By contrast, if homosexual couples are more promiscuous than heterosexual couples, then disease incidence rises in the male population, reducing this imbalance.

Another interesting aspect of our model is the finding of a backward bifurcation when there is disease induced mortality. This is particularly striking because it suggests that, once disease spread has taken hold, it can be very difficult to eradicate. In particular, when backward bifurcation occurs, identical conditions (e.g. same levels of mosquito control, same human sexual behavior) can lead to either disease extinction or endemicity depending on initial conditions. Consequently, once the system has gotten out of control, more aggressive management than would otherwise be necessary, may be required to reduce disease spread and return the system to a state below the bifurcation point. Another concerning aspect of backward bifurcation is that disease risk and/or incidence could increase suddenly with even small changes in management strategies. In particular, if the system lies beyond this bifurcation point, then even a slight decrease in mosquito control efforts, or a slight increase in promiscuity could move the system into a regime where only the endemic state is stable, and disease incidence levels are already quite high.

Although our model has explored a range of aspects associated with the interplay between vector and sexual transmission of ZIKV, including the role of heterosexual versus homosexual spread, and the implications of unidirectional transmission during heterosexual encounters, a number of additional biological aspects remain to be explored. As an example, current evidence suggests that Zika can remain in semen for several months, even though it is cleared from the blood within weeks. This suggests that the different modes of spread may have very different timescales. In particular, the slow timescale associated with clearance from sexual organs could increase the importance of this mode of spread with respect to its role in overall disease dynamics. Likewise, although we have considered both heterosexual and homosexual partnerships, incorporation of additional social structure could be informative. Indeed, in other sexually transmitted diseases, it is known that preferential mixing can dramatically influence disease dynamics. These, and other extensions, though they would make our model far less tractable from a mathematical perspective, could be important to consider from a biological standpoint.

In this paper, we formulated and analyzed a system of ordinary differential equations for transmission dynamics of Zika virus. Some of our theoretical and epidemiological findings are summarized below:

(i). The model disease free equilibrium is locally and globally asymptotically stable when the associated reproduction $\left(\mathcal{R}_{F}\right)$ is less than unity and unstable otherwise;

(ii). The model exhibits in the presence of disease induced mortality the phenomenon of backward bifurcation, where the stable disease-free co-exists with a stable endemic equilibrium, when the associated reproduction number is less than unity;

(iii). The model has a unique endemic equilibria for the system with heterosexual only and 
homosexual male only couples; these equilibria are locally asymptotically stable when their respective reproduction numbers are greater than unity;

(iv). Risky behavior among the male population increases the number of infected individuals in that population;

(v). The sexual transmission route contributes significantly to the disease burden in the community.

\section{Acknowledgments}

This research was supported by the Strategic Environmental Research and Development Program under grant RC-2639. One of the author, FBA, has additional support from University of Kansas New Faculty grant and WFF, has further support from the US National Science Foundation under grant DMS-1225917.

\section{References}

[1] Agusto, F.B., Gumel, A.B. (2013). Qualitative dynamics of lowly- and highly-pathogenic avian influenza strains. Math. Biosci. 243(2): 147-162.

[2] Agusto, F.B., Gumel, A.B., Lenhart, S. and Odoi, A. (2011). Mathematical Analysis of the Transmission Dynamics of Bovine Tuberculosis Model. Journal of Mathematical Methods in Applied Sciences. 34: 1873-1887.

[3] Agusto, F.B. and Ogunye, O.R. (2010). Avian Influenza Optimal Seasonal Vaccination Strategy. The Anziam Journal. 51: 394-405.

[4] Anderson, R. M. and May, R. (1991). Infectious Diseases of Humans, Oxford University Press, New York.

[5] Atkinson, Barry, et al. (2016). Detection of Zika virus in semen. Emerging infectious diseases. 22(5): 940 .

[6] Bewick, S., Fagan, W. Calabrese, J. and Agusto, F. (2016). Zika Virus: Endemic Versus Epidemic Dynamics and Implications for Disease Spread in the Americas. Submitted.

[7] Brauer, F. (2004). Backward bifurcations in simple vaccination models. J. Math. Anal. Appl. 298(2): 418-431.

[8] Brauer, F. and van den Driessche, P. (2001). Models for transmission of disease with immigration of infectives, Math. Biosc. 171: 143-154.

[9] Brent, C., Dunn, A., Savage, H., Faraji, A., et al. (2016). Preliminary Findings from an Investigation of Zika Virus Infection in a Patient with No Known Risk Factors Utah, 2016. PreventionMorbidity and Mortality Weekly Report. Early Release. 65(13): 1-2. Peterson, D., Christensen, K., Dimond, M., Staples, J.E., Nakashima, A.

[10] Cao-Lormeau, Van-Mai, et al. (2016). Guillain-Barre Syndrome outbreak associated with Zika virus infection in French Polynesia: a case-control study. The Lancet. 387(10027): 15311539 . 
[11] Carr, J. Applications of Centre Manifold Theory. Springer-Verlag, New York, 1981.

[12] Castillo-Chavez, C., Blower, S., van den Driessche, P., Kirschner, D., and Yakubu, A.A. (2002). Mathematical Approaches for Emerging and Reemerging Infectious Diseases, Springer-Verlag, New York.

[13] Castillo-Chavez C. and Song, B. (2004). Dynamical models of tuberculosis and their applications. Math. Biosci. Engrg. 1(2): 361-404.

[14] Centers for Disease Control and Prevention. (2016). All Countries \& Territories with Active Zika Virus Transmission. https://www.cdc.gov/zika/geo/active-countries.html. Accessed on June 20, 2016.

[15] Centers for Disease Control and Prevention. (2016). Zika virus.

http://www.cdc.gov/zika/symptoms/index.html. Accessed June 2, 2016.

[16] Coelho, Flávio Codeo, et al. (2016). Sexual transmission causes a marked increase in the incidence of Zika in women in Rio de Janeiro, Brazil. bioRxiv. 055459.

[17] Deckard, D.T., Chung, W.M., Brooks, J.T., et al. (2016). Male-to-Male Sexual Transmission of Zika Virus Texas, January 2016, Morbidity and Mortality Weekly Report. 372(65): 14.

[18] Dick, G.W.A., Kitchen, S.F. and Haddow. A.J. (1952). Zika virus (I). Isolations and serological specificity. Transactions of the Royal Society of Tropical Medicine and Hygiene. 46(5): 509-520.

[19] Diekmann, O., Heesterbeek, J.A.P. and Metz, J.A.P. (1990). On the definition and computation of the basic reproduction ratio $R_{0}$ in models for infectious diseases in heterogeneous populations. J. Math. Biol. 28: 503-522.

[20] Dirlikov, E., Ryff, K.R., Torres-Aponte, J., et al. (2016). Update: Ongoing Zika Virus Transmission - Puerto Rico, November 1, 2015-April 14 2016. MMWR. 65(17): 451-455.

[21] D'Ortenzio, Eric, et al. (2016). Evidence of sexual transmission of Zika virus. New England Journal of Medicine. 374(22): 2195-2198.

[22] Dubrulle, M. Mousson, L. Moutailler, S. Vazeille, M. and Failloux, A.B. (2009). Chikungunya virus and Aedes mosquitoes: saliva is infectious as soon as two days after oral infection. PLoS One. 4(6): e5895.

[23] Duffy, M.R., Chen, T.H., Hancock, W.T., et al. (2009). Zika virus outbreak on Yap Island, Federated States of Micronesia. New England Journal of Medicine. 360(24): 2536-2543.

[24] Dumont, Y. Chiroleu, F. and Domerg, C. (2008). On a temporal model for the Chikungunya disease: Modeling, theory and numerics. Mathematical Biosciences. 213(1): 80-91.

[25] Dumont Y. and Chiroleu. F. (2010). Vector control for the Chikungunya disease. Mathematical Biosciences and Engineering. 7(2): 315-348.

[26] Dushoff, J. Wenzhang, H. and Castillo-Chavez, C. (1998). Backwards bifurcations and catastrophe in simple models of fatal diseases. J. Math. Biol. 36: 227-248. 
[27] Foy, B.D., Kobylinski, K.C., Chilson Foy, J.L., et al. (2011). Probable non-vector-borne transmission of Zika virus, Colorado, USA. Emerg. Infect. Dis. 17(5): 880-882.

[28] Gray, Ronald H., et al. (2001). Probability of HIV-1 transmission per coital act in monogamous, heterosexual, HIV-1-discordant couples in Rakai, Uganda. The Lancet. 357(9263): $1149-1153$.

[29] Haddow, Andrew D., et al. (2012). Genetic characterization of Zika virus strains: geographic expansion of the Asian lineage. PLoS Negl. Trop. Dis. 6(2): e1477.

[30] Haddow, A.J., et al. (1964). Twelve isolations of Zika virus from Aedes (Stegomyia) africanus (Theobald) taken in and above a Uganda forest. Bulletin of the World Health Organization. 31(1): 57 .

[31] Hethcote, H.W. (2000). The mathematics of infectious diseases, SIAM Rev. 42(4): 599-653.

[32] Hills, S.L. Russell, K., Hennessey, M., et al. (2016). Transmission of Zika Virus Through Sexual Contact with Travelers to Areas of Ongoing Transmission Continental United States. MMWR. 65(8): 215-216.

[33] Hillin, T. (2014). This Is How Often Married People Are Having Sex. http://www.huffingtonpost.com/2014/07/17/married-sex_n_5596185.html. Accessed June 27, 2016.

[34] Jeffries, C. Klee, V. and Van Den Driessche, P. (1977). When Is A Matrix Sign Stable? Can. J. Math. 29(2): 315-326.

[35] Karimi, F. (2016). 3 deaths linked to Zika virus in Venezuela. Cable News Network. http://www.cnn.com/2016/02/12/health/venezuela-ziva-virus-venezeuela/. Accessed May 2, 2016.

[36] Lakshmikantham, V., Leela, S. and Martynyuk, A.A. (1989). Stability Analysis of Nonlinear Systems. Marcel Dekker, Inc., New York and Basel.

[37] Lyons, Anthony, Marian Pitts, and Jeffrey Grierson. (2013). Versatility and HIV vulnerability: Patterns of insertive and receptive anal sex in a national sample of older Australian gay men. AIDS and Behavior. 17(4): 1370-1377.

[38] Manore, C., Hickmann, J., Xu, S., Wearing, H., and Hyman, J. (2014). Comparing dengue and chikungunya emergence and endemic Transmission in A. aegypti and A. albopictus. Journal of Theoretical Biology. 356(7): 174-191.

[39] Mansuy, Jean Michel, et al. (2016). Zika virus: high infectious viral load in semen, a new sexually transmitted pathogen. Lancet Infect Dis. 16(405): 00138-00139.

[40] Marchette, N.J., Garcia, R. and Rudnick. A. (1969). Isolation of Zika virus from Aedes aegypti mosquitoes in Malaysia. American Journal of Tropical Medicine and Hygiene. 18(3): 411-415.

[41] Marcondes, Carlos Brisola, and Maria de Fitima Freire de Ximenes (2016). Zika virus in Brazil and the danger of infestation by Aedes (Stegomyia) mosquitoes. Revista da Sociedade Brasileira de Medicina Tropical. 49(1): 4-10. 
[42] McCarthy, M. (2016). Zika virus was transmitted by sexual contact in Texas, health officials report. BMJ. 352: i720.

[43] Mlakar, Jernej, et al. (2016). Zika virus associated with microcephaly. New England Journal of Medicine 374(10): 951-958.

[44] Moulay, D., Aziz-Alaoui, M.A. and Cadivel, M. (2011). The chikungunya disease: modeling, vector and transmission global dynamics. Mathematical Biosciences. 229(1): 50-63..

[45] Musso, D., Roche, C., Robin, E., Nhan, T., Teissier, A., and Cao-Lormeau, V. M. (2015). Potential sexual transmission of Zika virus. Emerging infectious diseases. 21(2): 359.

[46] Newton, E.A.C and Reiter, P. (1992). A model of the transmission of dengue fever with an evaluation of the impact of ultra-low volume (ULV) insecticide applications on dengue epidemics. American Journal of Tropical Medicine and Hygiene. 47: 709-720.

[47] Oehler, E., et al. (2014). Zika virus infection complicated by Guillain-Barre syndrome-case report, French Polynesia, December 2013. Euro Surveill. 19(9): 20720.

[48] Oliveira Melo, A.S., et al. (2016). Zika virus intrauterine infection causes fetal brain abnormality and microcephaly: tip of the iceberg?. Ultrasound in Obstetrics \& Gynecology. 47(1): $6-7$.

[49] Olson, J.G., and Ksiazek, T.G. (1981). Zika virus, a cause of fever in Central Java, Indonesia. Transactions of the Royal Society of Tropical Medicine and Hygiene. 75(3): 389-393.

[50] Paupy, C., Ollomo, B., Kamgang, B., et al. (2010). Comparative role of Aedes albopictus and Aedes aegypti in the emergence of dengue and chikungunya in Central Africa. Vector-Borne and Zoonotic Diseases. 10(3): 259-266.

[51] Petersen, L.R., et al. (1992). No Evidence for Female-to-Female HIV Transmission Among 960,000 Female Blood Donors. JAIDS Journal of Acquired Immune Deficiency Syndromes. 5(9): 853-855.

[52] Poletti, P., Messeri, G., Ajelli, M., et al. (2011). Transmission Potential of Chikungunya Virus and Control Measures: The Case of Italy. PLoS ONE. 6(5): e18860.

[53] Putnam, J.L. and Scott, T.W. (1995). Blood feeding behavior of dengue-2 virus-infected Aedes aegypti. American Journal of Tropical Medicine and Hygiene. 55: 225-227.

[54] Samarasekera, Udani, and Marcia Triunfol. (2016). Concern over Zika virus grips the world. The Lancet. $387(10018)$ : 521-524.

[55] Sarmiento-Ospina, A., Vásquez-Serna, H., Jimenez-Canizales, C.E., et al. (2016). Zika virus associated deaths in Colombia. Lancet Infect Dis. 16: 523-524.

[56] Sebastian, M.R., Lodha, R. and Kabra, S.K. (2009). Chikungunya infection in children. Indian Journal of Pediatrics. 76(2): 185-189.

[57] Sheppard, P.M., Macdonald, W.M., Tonn, R.J. and Grabs. B. (1969). The dynamics of an adult population of Aedes aegypti in relation to dengue haemorrhagic fever in Bangkok. Journal of Animal Ecology. 38: 661-701. 
[58] Smith H.L. and Waltman. P. The Theory of the Chemostat. Cambridge University Press, 1995.

[59] Traynor, Kate. (2016). Public health officials alarmed at spread of Zika virus. Am. J. Health Syst. Pharm. 73: 508-509.

[60] Trpis, M. and Haussermann, W. (1986). Dispersal and other population parameters of Aedes aegypti in an African village and their possible significance in epidemiology of vector-borne diseases. American Journal of Tropical Medicine and Hygiene. 35: 1263-1279.

[61] Trpis, M. Haussermann, W. and Craig, G.B. (1995). Estimates of population size, dispersal, and longevity of domestic Aedes aegypti by mark-release-recapture in the village of Shauri Moyo in eastern Kenya. Journal of Medical Entomology. 32: 27-33.

[62] Turell, M.J., Beaman, J.R. and Tammariello, R.F. (1992). Susceptibility of selected strains of Aedes aegypti and Aedes albopictus (Diptera: Culicidae) to chikungunya virus. Journal of Medical Entomology. 29(1): 49-53.

[63] van den Driessche, P. and Watmough, J. (2002). Reproduction numbers and sub-threshold endemic equilibria for compartmental models of disease transmission. Math. Biosci. 180: $29-48$.

[64] Venturi, G., et al. (2016). An autochthonous case of Zika due to possible sexual transmission, Florence, Italy, 2014. Euro Surveill. 21(8): 30148.

[65] Wikipedia (2015). List of sovereign states and dependent territories by birth rate. https://en.wikipedia.org/wiki/List_of_sovereign_states_and_dependent_ territories_by_birth_rate. Accessed February 1, 2016.

[66] World Health Organization (2016). Microcephaly Brazil: Disease Outbreak News. http://www.who.int/csr/don/8-january-2016-brazil-microcephaly/en/. Accessed February 1, 2016.

[67] Wonham, Marjorie J., et al. (2006). Transmission assumptions generate conflicting predictions in hostvector disease models: a case study in West Nile virus. Ecology letters. 9(6): 706-725.

[68] Zanluca, Camila, et al. (2015). First report of autochthonous transmission of Zika virus in Brazil. Memórias do Instituto Oswaldo Cruz. 110(4): 569-572.

\section{A Appendix A: Proof of Lemma 1}

Proof. Let $t_{1}=\sup \{t>0: F(t)>0\}$. Thus, $t_{1}>0$. Then it follows from the first equation of the Zika model (2.5) that

$$
\frac{d S_{F}}{d t}=\frac{\Pi_{H}}{2}-\left[\lambda_{H}\left(I_{V}, N_{H}\right) S_{F}(t)+\lambda_{F M}\left(I_{M}, N_{F}\right) S_{F}(t)-\mu_{F} S_{F}(t)\right.
$$

can be re-written as 